\title{
DETERMINISMO Y LIBERTAD EN LA FILOSOFÍA NATURAL DE DESCARTES
}

\author{
Laura Benítez*
}

RESUMEN: Se busca mostrar diversas tensiones en la filosofia natural de Descartes al introducir una propuesta desarrollista. El filósofo francés hereda el problema teológico de la potentia absoluta Dei y la potentia ordinata Dei. Las preocupaciones cartesianas se ven envueltas en la reflexión neoplatónica sobre la infinitud: su perspectiva acerca de Dios podría caracterizarse como potentia infinita Dei.

En cuanto a la libertad y el determinismo, si bien en la inmutabilidad de Dios se funda la causalidad del mundo en última instancia, el único hecho necesario es que Dios no altera las propiedades esenciales de la materia, pero la diversidad de cuerpos y movimientos son efectos accidentales. Así, si bien Dios conserva el universo del mismo modo que lo ha creado, no las partes materiales en el mismo estado. En suma, el universo cartesiano resulta a la vez necesario y contingente.

PALABRAS ClAVE: Necesidad, contingencia, potencia absoluta, potencia ordenada y potencia infinita, modo y estado.
ABSTRACT: In this article, we will observe that there are different tendencies in Descartes' natural philosophy when introducing a progressive proposal. This French philosopher inherits the theological dilemma of potentia absoluta Dei and potentia ordinata Dei. These Cartesians worries are surrounded by neoplatonic reflexions on infiniteness: his perspective about God could be designated as potentia infinita Dei.

As for freedom and determinism, the world's causality is derived from God's immutable nature, thus preserving only the essential properties of matter but not those accidental effects, such as the diversity of shapes and movements. Therefore, even when the universe is preserved in its mode, its material components do not remain in the same state. In other words, the Cartesian universe is both necessary and contingent.

KEYWORDS: Necessity, contingency, absolute power, ordained and infinite power, mode and state.

* Instituto de Investigaciones Filosóficas, UNAM. 


\section{DETERMINISMO Y LIBERTAD EN LA FILOSOFÍA NATURAL DE DESCARTES}

\section{Introducción}

El tema del mundo natural está dominado, en Descartes, por dos grandes preocupaciones que crean diversas tensiones al interior de la teoría. Una preocupación es lograr para la filosofía natural un lenguaje propio e independiente del dictum teológico; la otra, es no hallar mejor fundamento que el ser divino para entender la génesis y desarrollo del mundo. En mi opinión, la mejor que Descartes encontró para solucionar este conflicto fue la "vía cusana", que separa la infinitud de Dios de la ilimitación del universo. Con ello, Descartes buscó circunscribir los procesos espaciales, temporales y numéricos del universo, a su diversidad de entidades, sucesos, cambios, divisiones, agregados, etc. En tales procesos, ciertamente, no encontramos límite (como es el caso de la división de la materia o la cantidad de las estrellas), pero no por ello hablamos de infinitud, sino de ilimitación por dos razones: una epistemológica, porque desconocemos si el proceso de división de la materia o de agregación de estrellas tienen un límite; y, la otra, ontológica porque, aunque de facto no hubiera un límite, el mero hecho de ser procesos nos habla de su infinitud potencial por lo cual, en el caso del mundo, no podemos hablar de infinitud actual. ${ }^{1}$ Así, Descartes

\footnotetext{
${ }^{1}$ Descartes, Princ. I, 26 AT IX, 36.
} 
propone como lenguaje propio de la filosofía natural el de la ilimitación, con todas las dificultades que ello pudiera acarrear, incluidas naturalmente la acusación de materialismo y de panteísmo.

El otro gran problema es el de la génesis y desarrollo del universo. Sobre la generación del universo, Descartes no tiene dudas: Dios todopoderoso ha generado el universo, pero ello directamente es una verdad de fe y sobre la omnipotencia divina el filósofo no desea disputar con los teólogos. Ellos han preguntado en una discusión que viene desde la Edad Media si Dios pudo haber hecho el mundo de otra manera y con otras leyes o, si Dios, por su omnipotencia puede dirigirse hacia la nada, etc. Descartes se contenta con declarar que él no disputa sobre cuestiones de fe que están absolutamente por encima de la razón. Pero el filósofo no se queda tranquilo pues en su ambiente intelectual todavía gravita el conflicto de la potentia ordinata Dei y la potentia absoluta Dei. No obstante, encuentra una importante salida cuando recurre a la infinitud de Dios alejándose de la tradición escolástica y acogiéndose a una reflexión platónica intelectualista. En efecto, la vía de la infinitud subraya la simplicidad y unidad de la divinidad, no la analiza por aspectos ni contrapone unos a otros sino que, en una visión inte- gradora, concibe que la creación del ser es la perfección de Dios. Así, la polémica escolástica se limita más a las Meditaciones en tanto que los Principios de la filosofía se asientan más sobre la reflexión intelectualista de la infinitud divina. A primera vista, la generación y regulación del mundo, al provenir de un ser necesario, no puede sino reflejar esa inmutabilidad por lo que los procesos del mundo estarían irremediablemente determinados. Esta lucha entre la necesidad y la contingencia del universo se manifiesta en las disputas medievales que Descartes retomará en su caracterización del mundo.

\section{Las preocupaciones cartesianas en torno a la omnipotencia divina}

Según nos informa Thierry Gontier, ${ }^{2}$ la oposición entre lo que Dios puede hacer y lo que quiere hacer, se manifiesta ya en las Sentencias de Pedro Lombardo y fue discutida por la mayoría de sus comentaristas entre los cuales sobresale Duns Escoto. De hecho, la omnipotencia divina, se asienta como dogma de fe cuando se reconoce a Dios como padre creador de todas las cosas pero, como todo dogma, éste se muestra inaccesible a la razón. Sin embargo, la radi-

${ }^{2}$ Thierry Gontier, Descartes et la causa sui, 2005, París, Vrin. 
calidad del dogma cristiano estriba en que no sólo se reconoce la fuerza creadora de Dios, sino su absoluta e irrestricta libertad. Ello significa que Dios no sólo crea "bajo el modo de la necesidad", sino que es "una voluntad capaz de causar en el mundo lo contingente". ${ }^{3}$ Para Duns Escoto, los filósofos limitan a Dios cuando consideran que todo lo que produce es necesario pues no toman en cuenta su potencia absoluta. Así, la libertad de Dios, no sólo es independencia ontológica frente al universo, sino capacidad irrestricta de causación y de elección.

Frente a la potentia absoluta Dei se levanta la potentia ordinata Dei pero, en general, en los autores no se trata tanto de una oposición cuanto de una distinción en el ámbito teológico, que obedece a la forma escolástica de plantear los atributos divinos por separado, ya que la misericordia, la sabiduría o la bondad no se deducen directamente del libre arbitrio divino, sino que lo complementan. De ninguna manera podemos pensar que en Dios la voluntad está en lucha con el intelecto, en el sentido de que Dios esté atado por las propias leyes con que ordena al universo o que no tuviera la posibilidad de rebasar los principios lógicos; simplemente, para decirlo con Occam, entre la potentia absoluta Dei y la potentia ordinata Dei no hay

${ }^{3}$ Ibid., pp. 52-3. Gontier cita a Duns Escoto. sino una distinción mínima de razón. ${ }^{4}$ Se trata pues de diversos aspectos de la divinidad que en su conjunto rebasan nuestra comprensión.

Empero, las distinciones escolásticas no fueron las únicas que preocuparon a Descartes. De hecho, su visión del creador y el universo creado se ve frecuentemente traspasada por la reflexión neoplatónica de la infinitud. Conviene advertir, sin embargo, que a diferencia de las propuestas teológicas de la escolástica: "el horizonte teológico del neoplatonismo es [como nos dice T. Gontier] eminentemente especulativo". ${ }^{5}$ Ya Duns Escoto advertía que los filósofos ven a Dios como ser infinito y no como Dios, esto es, como ser omnipotente. De hecho, para Plotino, violar los principios lógicos es más un signo de impotencia que de poder. ${ }^{6}$

En el neoplatonismo, se insiste en la simplicidad divina y todos los atributos se vinculan sin distinción en el ser infinito de Dios y es la debilidad de nuestro espíritu la que nos impide captar esa simplicidad, a la cual no se accede racionalmente, sino en el esfuerzo de simplificación del alma que tiende a lo uno.

En cuanto a Descartes, encontramos tanto la terminología de la “omni-

\footnotetext{
${ }^{4} I b i d .$, p. 54.

${ }^{5}$ Ibid., p. 55.

${ }^{6}$ Eneada VI, 8, $3{ }^{\mathrm{a}}$.
} 
potencia divina" como la de la "potencia infinita". Aquí lo importante es saber cómo asume nuestro autor tales propuestas. Si bien, en las $\mathrm{Me}$ ditaciones en general asume el dogma de la omnipotencia, en las Respuestas y en la Correspondencia, introduce el matiz de que la omnipotencia divina rebasa la razón y no es factible una comprensión filosófica de ello. En general, su posición es de cautela y de no pronunciarse, por ejemplo, sobre si Dios puede o no hacer algo contradictorio, cuando Arnauld se lo pregunta.

Así, Descartes encuentra menos dificultad para referirse a la divinidad desde el discurso neoplatónico de la infinitud. Prudentemente escotista, deja que el teólogo hable de la potentia absoluta Dei. Por ello, es más claramente afín a la idea de que la no realización, por parte de Dios, de los imposibles lógicos u ontológicos, no es señal de impotencia divina sino, por el contrario, es un signo de la infinitud de su poder. Un claro ejemplo de ello, en las Meditaciones, es el recorrido de la duda hiperbólica. Si Dios lo quisiera podría engañarme siempre, pero su bondad limita extrínsecamente esta libertad absoluta. La conclusión podría ser que por bondad puede engañarme algunas veces, pero Descartes no quiere entrar en la polémica del "derecho a mentir" que ejercen padres, maestros o médicos, por el bien de sus hijos, pupilos o pacientes. Así, en las $\mathrm{Me-}$ ditaciones tercera y cuarta, Descartes deja en claro que su perspectiva es la de la idea de potentia infinita Dei. Ésta, señala la capacidad de producción del ser y excluye el no ser, por lo que cualquier posibilidad negativa que incluye el no ser, como es el caso del engaño, es contrario a la capacidad creadora infinita de Dios. ${ }^{7}$

\section{Conocimiento pleno o completo sobre Dios y el mundo}

Descartes sostiene la propuesta epistemológica de que no tenemos ideas enteramente perfectas acerca de ninguna cosa, mucho menos de Dios; con lo que sí contamos es con ideas adecuadas, esto es, concepciones plenas o completas sobre el ser supremo, las cuales nos ofrecen, no sólo un nivel de certeza o seguridad psicológica, sino que se muestran como evidentes, claras y distintas a nuestro entendimiento.

Desde un punto de vista puramente filosófico, Descartes puede afirmar que, siendo la idea de Dios una idea

${ }^{7}$ Ibid., p. 67. Gontier concluye: "En suma, Descartes invoca a menudo el poder infinito de Dios, pero no se compromete con la omnipotencia en sentido occamiano, si lo utiliza lo hace en sentido retórico". 
innata, por ende, clara y distinta, nosotros no advertimos positivamente ningún límite a sus perfecciones, por lo cual comprender esta idea es, a partir del análisis intelectual, adjudicarle la perfección infinita. En ello consiste nuestra idea adecuada o plena de Dios. ${ }^{8}$

En el caso del mundo, el problema epistemológico se vuelve más complejo. En primer lugar, no contamos con ideas innatas de los cuerpos, sino tan sólo de algunas de sus propiedades como la extensión. De los fenómenos o apariencias sólo tenemos ideas adventicias. De este modo, hay que combinar, para el conocimiento del mundo natural, algunas ideas innatas matemáticas, geométricas y físicas, con ideas adventicias, esto es, ideas sensibles recurrentes. A partir de ello, debemos establecer los parámetros de las regularidades fenoménicas, para contar con algunas teorías razonables acerca del mundo y su comportamiento.

Nuestros principios o leyes son nuestras ideas adecuadas o completas acerca del mundo y están muy lejos de ser ideas enteramente perfectas, pues aunque sean ideas claras

${ }^{8}$ René Descartes, Méditations, A/T IX, 172 [Oeuvres de Descartes, 1996, Paris, Vrin, Librairie Philosophique, ed. Charles Adam \& Paul Tannery]. Descartes le aclara a Arnauld, en las Respuestas a las Terceras Objeciones, por qué tenemos un conocimiento pleno y completo, aunque no perfecto de Dios. y distintas, nuestra razón se ve limitada para aplicarlas a una ilimitada cantidad de objetos del universo.

Descartes distingue cuidadosamente entre infinitud e ilimitación, no sólo por la distinta forma en que conocemos a Dios y al mundo, sino porque de entrada les atribuye muy distinta naturaleza.

La razón para usar el término "indefinido" más que el término "infinito", con relación a estas cosas [las cosas creadas] es, en primer lugar, reservar el término "infinito" únicamente para Dios. Porque únicamente en el caso de Dios no sólo no reconocemos ningún límite en ningún respecto, sino que nuestro entendimiento positivamente nos dice que no lo hay. En segundo lugar, en el caso de las otras cosas, nuestro entendimiento no nos dice, positivamente, que carecen de límites en algún aspecto, sino que meramente reconocemos, de modo negativo, que cualquier límite que pudieran tener no puede ser descubierto por nosotros. ${ }^{9}$

Aunque esta propuesta se aloja en el esquema neoplatónico de la infinitud, es claro que Descartes enfatiza la diferencia ontológica entre Dios y el mundo, pues si bien el intelecto reconoce la infinitud positiva de Dios, no sabe si los procesos del uni-

${ }^{9}$ Principios..., I, 27, AT IX, 37. 
verso son, de facto, ilimitados. Esta aseveración de los Principios se verá matizada en sus respuestas a las cartas de Henry More. Allí, sin alterar la idea de creador y creación, esencialmente de infinitud divina y de ilimitación del universo, acepta que los procesos del mundo pueden ser comprendidos como positivamente ilimitados. Así, a partir del argumento epistemológico, Descartes dice que no puede saberse si el universo tiene o no límites. Pero, al sustanciar el argumento ontológico: "está en conflicto con mi posición atribuirle algún límite al mundo [...] La razón por la que digo que el mundo es indeterminado o indefinido es que no puedo descubrir límites en él. ${ }^{10}$

Aunque, a primera vista, se trata de un argumento débil, a saber, "El mundo es ilimitado porque no puedo encontrarle límites", en realidad Descartes trata de subrayar precisamente la naturaleza ontológica del mundo como inacabado, procesual, potencial, etc. More tergiversa a Descartes. Si el mundo es indefinido per se entonces es infinito, ergo es semejante a Dios, ergo Descartes es un ateo (pues para esos autores panteísta y ateo eran los mismo).

\section{Libertad y determinismo en el mundo natural}

Una vez asentadas la omnipotencia y la infinitud divinas, así como su trascendencia frente al mundo creado, Descartes avanza en el problema de la causalidad. En efecto, postula la inmutabilidad de Dios en el sentido de causa que produce siempre el mismo efecto, puesto que sólo la regularidad inalterable de la relación causal puede darle sustento al conocimiento de la naturaleza como universal y necesario.

La propuesta es doble, por un lado, Dios crea del universo, o más específicamente, toda la materia que existe a nuestro alrededor, concebida esencialmente como extensión indefinida; por otro, Dios la dota de movimiento.

En suma, si Dios es infinitamente perfecto, Dios es inmutable y ¿qué mejor fundamento para la relación causal puede proponerse? Dice Descartes:

Porque, ¿cuál fundamento más firme y más sólido puede uno encontrar para establecer una verdad, incluso queriendo escoger a capricho, que el tomar la firmeza misma y la inmutabilidad que está en Dios? ${ }^{11}$

La inmutabilidad de Dios garantiza la permanencia de la misma can-

\footnotetext{
${ }^{11}$ René Descartes, Le Monde..., AT XI, 439.
} 
tidad de materia y de movimiento en el universo, pero no impide el cambio y la diversificación de los cuerpos. Dios inmutable garantiza, al ser su acción idéntica, la conservación del mundo creado, pero con las tendencias y disposiciones que ha puesto en la materia. Así, una vez dotada de movimiento y regulada por las leyes con las que la ha dotado, éstas darán cuenta inmediata de los cambios que sufre.

Descartes aspira a un conocimiento bien fundado y evidente del mundo natural; para ello, recurre a la inmutabilidad de Dios en la cual puede fundar, en última instancia, la causalidad del mundo, pero encuentra que en el mundo el único hecho necesario es que Dios no altera las propiedades esenciales de la materia. La diversidad de cuerpos y movimientos son efectos "accidentales" cuya variedad obliga a decir:

\section{En el mundo no todo efecto es necesario}

2. Además de los principios necesarios que rigen la naturaleza, pueden establecerse leyes particulares, fundadas en regularidades contingentes.

3. Los primeros dan cuenta del modo en que Dios conserva la sustancia del mundo, siempre como extensión en largo, ancho y profundo, con tendencia al movimiento rectilíneo.
4. Las segundas explican los diversos estados que la naturaleza adquiere, como el reposo, el movimiento, el cambio de figura, de tamaño y de lugar, el movimiento circular, etc.

Esta es la forma en que Descartes buscó conjugar determinismo y contingencia en el mundo natural.
A continuación, haciendo un repaso de los objetos que se han presentado a mis sentidos, me atrevo a decir que no he percibido ninguna cosa que no pudiese explicar muy fácilmente a través de los principios que he encon- trado. Pero es también necesario que afirme que el poder de la naturaleza es tan amplio y tan vasto y que estos principios son tan simples y tan ge- nerales, que no percibo casi ningún efecto particular que no conozca de antemano que puede ser dedu- cido de muy diversas formas y mi mayor dificultad es comúnmente de cuál de estas formas depende. ${ }^{12}$

A la vez que ilumina un aspecto epistemológico fundamental, a saber, que no se puede construir la ciencia deductivamente a partir de los primeros principios, sin recurrir a la observación de los efectos particulares, y después elevarnos inductivamente a los principios generales, al mismo tiempo nos habla del poder diversi-

\footnotetext{
${ }^{12}$ René Descartes, Discurso..., AT VI, 63.
} 
ficador de la naturaleza, casi opuesto al poder de la simplicidad divina. Esta cuestión y otras similares hicieron pensar a algunos comentaristas, por ejemplo a Bernard Williams, que Descartes, en las obras tempranas, se inclina por un esquema desarrollista, esto es, una vez creada por Dios la naturaleza se desarrolla contingentemente con la única restricción de ciertas leyes básicas. Ello parece, sin embargo, contradecirse con cierto esquema de "creación continua" que Descartes utiliza para explicar la temporalidad y que mencionaré brevemente más adelante.

En suma, a pesar de que Dios, como causa eficiente última, ha dictado los principios que rigen al universo, esto es, las leyes del movimiento, la diversidad y cambio que percibimos en el mundo natural, son el efecto diversificado de estas mismas leyes, que en cuanto causas inmediatas, explican sus efectos o procesos.

Es la forma en que Descartes conjuga libertad y necesidad o determinismo y contingencia en el mundo natural.

\section{El principio de permanencia y las leyes del cambio}

Si Dios crea el mundo y dicta sus leyes es de esperarse que el mundo, dada la naturaleza inmutable de su creador, se comporte siempre de la misma manera, esto es, que el principio de orden esté siempre presente, pero ello no significa que el mundo no presente constantes cambios, fenómenos variados, etc. La pregunta es entonces, ¿qué es lo que Dios crea y por tanto qué es lo que conserva, dado el cambio constante observable en el universo?

Nuevamente, frente a la tradición religiosa, particularmente del Génesis, el mecanicismo cartesiano se muestra más directamente en el El mundo o tratado de la luz, divergente, en tanto que en el resto de sus escritos se pronuncia con cautela. Así nos dice $\mathrm{B}$. Williams que:

En la Conversación con Burman (V168-169 C.pp.36-37) Descartes menciona el proyecto de reconciliar su versión con el Génesis -o más bien, deberíamos decir, reconciliar el Génesis con su versión-dando una interpretación metafórica de la narración del Génesis. Dice que el asunto debería dejarse a los teólogos. ${ }^{13}$

La cuestión no es menor; en $E l$ mundo..., Descartes había propuesto claramente, de acuerdo con su corpuscularismo y su mecanicismo, un modelo desarrollista del universo, aunque dice que es un modelo "falso", una especie de mito, este "nuevo

${ }^{13}$ Bernard Williams, El proyecto de la investigación pura, 1995, México, UNAM, Instituto de Investigaciones Filosóficas, p. 273, nota 7. 
mundo" que describe, ya que entra en conflicto con las creencias religiosas. Pero más allá de la religión y la teología, Descartes sostiene en $E l$ Mundo... el principio de conservación referido a la cantidad total de movimiento en el universo y asociado con la potentia ordinata Dei. Después de decirnos que no hay fundamento más sólido para establecer la verdad que la inmutabilidad de Dios, exclama:

Luego estas dos reglas se siguen manifiestamente de ello, que Dios es inmutable y que, actuando siempre del mismo modo, produce siempre el mismo efecto. Porque suponiendo que ha puesto cierta cantidad de movimiento en toda la materia en general, desde el primer instante en que la ha creado, es necesario admitir que la conserva siempre así, o no creer que actúe siempre del mismo modo. ${ }^{14}$

La física cartesiana exhibe así uno de sus más importantes fundamentos metafísicos, la ley de permanencia relacionada directamente con la inmutabilidad de Dios, mediante la cual se asegura que no habrá cambios ni en las reglas, ni en las cantidades del juego mecánico del universo. Tal parecería, desde esta perspectiva, que Descartes se apoya únicamente en la absoluta racionali-

${ }^{14}$ Descartes, Le Monde, A/T XI,43. dad de Dios, pero ello sólo constituye un aspecto de la propuesta cartesiana. En efecto, Descartes también toma en cuenta los cambios en el mundo y esta interesante es trama la que me interesa destacar.

En el capítulo VII de El mundo, Descartes nos habla de las leyes de la naturaleza. Se trata, en efecto, de explicitar el principio de conservación. La naturaleza es la materia con todos los atributos de extensión, figura y movimiento que Descartes propone. Ahora bien, el asunto importante es que Dios la conserva siempre del mismo modo (la même façon) en que la ha creado, pero lejos de un determinismo necesario, Descartes nos propone su modelo desarrollista. Así, dice:

Porque únicamente de ello, de que continúa conservándola [Dios a la materia] se sigue de necesidad que debe haber muchos cambios en sus partes, los cuales, no pudiendo, me parece, ser atribuidos propiamente a la acción de Dios, puesto que Él no cambia en absoluto, los atribuyo a la Naturaleza; y las reglas a partir de las cuales se realizan estos cambios las llamo leyes de la naturaleza. ${ }^{15}$

El modelo desarrollista cartesiano es naturalmente contingente, puesto que si es verdad que hay un principio de conservación, también

\footnotetext{
${ }^{15}$ Ibid., A/T XI, 37.
} 
es verdad que hay unas reglas del cambio. ¿Cómo conciliar entonces la ley de permanencia con las reglas del cambio? O de otro modo, ¿cómo no ir en contra de la base metafísica de la física?

Descartes nos recuerda que todas las partes materiales desde que han sido creadas tienen diversos movimientos de manera que, desde que comenzaron a moverse, comenzaron también a cambiar y a diversificar sus movimientos. A estas alturas la pregunta es, ¿qué es lo que Dios finalmente conserva? Descartes nos responde:

Si Dios las conserva en adelante del mismo modo que las ha creado [a las partes materiales] no las conserva en el mismo estado; es decir, que Dios actuando siempre del mismo modo y, en consecuencia, produciendo siempre el mismo efecto en sustancia, se encuentra, como por accidente, mucha diversidad en este efecto. ${ }^{16}$

En suma, Dios conserva la misma cantidad de materia y de movimiento, pero la diversidad del universo deberá atribuirse más a las causas segundas, eso sí, sin olvidar que han sido dictadas por la Causa Primera para su buen funcionamiento $\mathrm{y}$ orden.

Porque Dios ha establecido tan maravillosamente estas leyes que, aun-

${ }^{16} \mathrm{Ibid}$., A/T XI, 38 (cursivas mías). que supongamos que no ha creado nada más de lo que he dicho [esto es, materia y movimiento] e incluso que no ponga en ello ningún orden y proporción, sino que componga con ello un caos, el más confuso y embrollado que los poetas pudieran describir; ellas [las leyes] son suficientes para hacer que las partes de este caos se desenreden ellas mismas y se dispongan en tan buen orden que tengan la forma de un mundo muy perfecto. ${ }^{17}$

El problema epistemológico es, ¿realmente podemos abarcar la diversidad de efectos y fenómenos, resultado de la diversificación de la materia, que es el espectáculo que nos ofrece el mundo natural? Aquí de nueva cuenta el contingentismo y el desarrollismo de la propuesta cartesiana se hacen manifiestos en los Principios...cuando Descartes declara:

Señalamos más arriba que los cuerpos que componen el universo están hechos de una misma materia, que es divisible en toda clase de partes y que está actualmente dividida en muchas que se mueven diversamente, y cuyos movimientos son de alguna manera circulares; y que hay siempre una misma cantidad de estos movimientos en el mundo; pero no hemos podido determinar de la misma manera, qué tan grandes son las partes en las cuales esta materia está dividida, ni cuál es la velocidad

${ }^{17}$ Ibid., A/T XI, 34-35. 
con la que se mueven, ni qué círculos describen. Porque estas cosas, habiendo podido ser ordenadas por Dios de una infinidad de maneras diversas, es únicamente por la experiencia y no por la fuerza del razonamiento que podemos saber cuál de todas ellas ha escogido. Es por lo que ahora somos libres de suponer la que queramos con tal de que todas las cosas que se deduzcan de ella estén de acuerdo con la experiencia. $^{18}$

De acuerdo con esto, la forma en que conocemos al mundo es doble: en su modo de ser, garantizado por la inmutabilidad y conservación divina, como materia extensa divisible y en constante movimiento, cuya cantidad no varía; en su estado actual, como diversidad de cuerpos con tamaños, formas y velocidades varias de las cuales podemos hipotéticamente suponer algún tipo de regularidad, que hemos de confrontar con la experiencia porque desconocemos de facto el camino que Dios señaló al desarrollo del universo hasta su estado actual. La potentia absoluta Dei se revela ahora como tres límites a nuestro conocimiento del mundo: 1. desconocemos las condiciones iniciales del desarrollo del universo; 2. desconocemos los patrones de regularidad de la producción de los diversos efectos; 3 . desco-

\footnotetext{
${ }^{18}$ Descartes, Principios..., A/T IX, III, 46.
}

nocemos absolutamente las razones de Dios. De esta manera, nuestro conocimiento del mundo no puede incluir causas finales en que se manifiesta la pretensión, desmedida y soberbia, de averiguar los designios a la divinidad. Tampoco podemos aseverar, con certeza absoluta, sino únicamente moral (diríamos hipotética) cuáles son los patrones en el comportamiento de los cuerpos en el universo; $y$, finalmente, las suposiciones cosmológicas cartesianas sobre las condiciones iniciales del universo oscilan entre el caos más confuso que cualquiera pueda suponer y la materia como sólido continuo, lo cual no constituye tanto una fortaleza como Descartes lo supone, sino una debilidad de la propuesta teórica, como lo señala B. Williams. ${ }^{19}$

Para finalizar este apartado quisiera referirme al problema del principio de concurrencia. En efecto, la más pura tradición escolástica consideraba que una cosa no puede permanecer si cesa la acción del agente, la cual no sólo se refiere a los efectos que le puedan sobrevenir, sino a su verdadero ser. ${ }^{20}$ Descartes considera que, la existencia de las cosas, pende de la concurrencia divina, no sólo en tanto creadas, sino en tanto

${ }^{19}$ Bernard Williams, Descartes: el proyecto de la investigación pura, 1995, México, UNAM, Instituto de Investigaciones Filosóficas, trad. Laura Benítez, cfr., cap. IX, pp. 275-9.

${ }^{20}$ Cfr. Aquino, Summa Theologiae, I, q. 104, art. 1 . 
subsistentes. Así, en el resumen de las Meditationes, señala, refiriéndose a la Segunda Meditación:

Primero, a fin de saber que generalmente todas las sustancias, es decir las cosas que no pueden existir sin ser creadas por Dios son, por su naturaleza incorruptibles, y no pueden jamás dejar de ser, si no son reducidas a la nada por este mismo Dios que les quiera negar su concurso ordinario. ${ }^{21}$

El problema es que no se trata nada más de permitir que las cosas continúen o no en la existencia, sino que Descartes se compromete con una tesis casi tan fuerte como la de Tomás de Aquino, como bien lo señala John Cottingham. ${ }^{22}$ En efecto, en la Meditación Tercera, Descartes comenta:

En efecto, es una cosa clara y evidente (para quienes consideran con atención la naturaleza del tiempo) que una sustancia, para ser conservada en todos los momentos de su duración, tiene necesidad del mismo poder y de la misma acción, que sería necesaria para producirla y crearla totalmente de nuevo si no lo estuviera todavía. De suerte que la luz natural nos hace ver claramente que

${ }^{21}$ Descartes, Meditations..., A/T IX, 10.

${ }^{22}$ John Cottingham, A Descartes Dictionary, 1993, Oxford, Blackwell Publishers, p. 40; cfr. Tomás de Aquino, op. cit., I, q. 104, art. 1. la conservación y la creación no difieren sino en relación con nuestra manera de pensar y no de hecho. ${ }^{23}$

La versión de la creación continua sólo en apariencia favorece una tesis racionalista fuerte de la potentia ordinata Dei, pero bien vista, lo que hace es acentuar la contingencia del universo, que pende de instante en instante de la voluntad del creador. Así, los principios cartesianos de orden, conservación y concurrencia parecen guardar un cierto equilibrio que le permite a Descartes acercar la razón a la voluntad divina.

\section{Conclusiones}

Como hemos visto, para Descartes la causa última o mediata del mundo es Dios, de quien tenemos un concepto adecuado. Al analizar la idea de Dios que poseemos de manera innata, este esfuerzo de reflexión intelectual nos lleva a la concepción positiva del ser infinito, cuya más perspicua manifestación de potencia se da siempre del lado de la creación del ser y del rechazo de la negación y el no ser.

Como vimos también, Descartes relega la disputa entre potentia absoluta Dei y potentia ordinata Dei al ámbito de la fe y al saber de los teólogos; sin embargo, no soslaya que

\footnotetext{
${ }^{23}$ Descartes, Meditations..., A/T IX, 39.
} 
se manifiesta una sabiduría en el plan divino para el universo y que su legalidad es necesaria, por lo que, en conjunto, reconoce la potencia ordenadora de Dios.

Con todo, Descartes especifica que la causación divina se asienta en su inmutabilidad. Es la inmutabilidad del ser infinito lo que garantiza que: "aunque la tierra y los cielos perecerán, es decir, cambiarán de aspecto, de todos modos el mundo, es decir, la materia de la que está compuesto, no perecerá jamás". ${ }^{24}$

Pero esta inmutabilidad y unicidad de la sustancia del mundo, i. e. la materia qua extensión, y la necesidad de sus principios, no impiden su diversificación en cuerpos distintos y distintos movimientos a partir de las leyes de la naturaleza como sus causas inmediatas.

Pero, ¿de ello podría inferirse que para Descartes Dios ha creado el mundo ab eterno, esto es, para que exista indefinidamente hacia el futuro? Ciertamente no, ya que la persistencia del mundo pende de la voluntad divina, puesto que el universo para Descartes carece de fuerza ínsita. De otra manera, la materia del universo no tiene en sí un conato que le permita perseverar en el ser, por lo que Dios, como su causa eficiente, debe, según Descartes, conservarlo de momento a momento. ¿Hemos regresado después de todo a la inquietud escolástica acerca de la potentia absoluta Dei? 